Check for updates

Cite this: RSC Adv., 2019, 9, 28541

Received 7th June 2019

Accepted 3rd September 2019

DOI: $10.1039 / c 9 r a 04286 e$

rsc.li/rsc-advances

\section{Ultrasound assisted one-step synthesis of AuaPt dendritic nanoparticles with enhanced NIR absorption for photothermal cancer therapy $\dagger$}

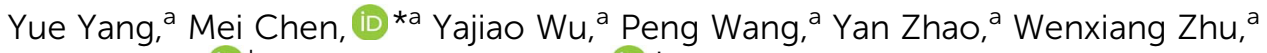 \\ Zhiling Song ${ }^{\mathrm{D}}{ }^{\mathrm{b}}$ and Xiao-Bing Zhang (D) *a
}

\begin{abstract}
Near-infrared (NIR) light-mediated non-invasive photothermal therapy (PTT) has attracted considerable attention for cancer treatment. Strong optical absorption located in the NIR region and high performance in converting light to heat should be emphasized for the development of ideal photothermal agents. In this report, Au@Pt bimetallic nanoparticles (Au@Pt NPs) with dendritic structure were synthesized through an ultrasound assisted one-step method in aqueous solution. The absorption of AuCPt NPs at $808 \mathrm{~nm}$ was obviously enhanced compared to that of $\mathrm{Au}$ NPs and could be easily manipulated via the amount of Pt NPs. AuaPt NPs exhibited excellent photostability with a photothermal conversion efficiency of $44.2 \%$, which is significantly higher than those in most reported studies. Au@Pt NPs with thiol PEG functionalization presented improved cellular killing capacity upon NIR laser irradiation. Moreover, the potential application of Au@Pt NPs was also investigated in xenograft tumor mouse model. Overall, the remarkable therapeutic characteristics of PEGylated Au@Pt NPs provide them with great potential for future cancer treatment.
\end{abstract}

\section{Introduction}

Photothermal therapy (PTT) exploiting near-infrared (NIR) light irradiation for cancer treatment has drawn widespread attention for its non-invasiveness, precise control and high therapeutic efficiency. ${ }^{1-7}$ Therefore, PTT is a promising candidate for treating diverse cancers compared with traditional cancer treatment technology possessing non-negligible disadvantages. In a typical PTT procedure, photothermal agents are first intravenously or intratumorally injected into mice bearing tumors. Subsequently, a NIR laser is specifically employed to irradiate them in the tumor sites to generate enough heat for the successful killing of cancer cells. To ensure maximized penetration in tissues and avoid the overheating effect in normal tissues, a NIR laser with wavelength ca. $800 \mathrm{~nm}$ situated in the first biological window (750-900 $\mathrm{nm}$ ) is usually chosen for PTT. ${ }^{8,9}$ Thus, the development of ideal photothermal agents with strong optical absorption in

\footnotetext{
${ }^{a}$ State Key Laboratory of Chemo/Biosensing and Chemometrics, College of Chemistry and Chemical Engineering, College of Materials Science and Engineering, Hunan University, Changsha 410082, PR China. E-mail: chenmei@hnu.edu.cn; xbzhang@ hnu.edu.cn

${ }^{b}$ Key Laboratory of Optic-electric Sensing and Analytical Chemistry for Life Science, $M O E$, College of Chemistry and Molecular Engineering, Qingdao University of Science and Technology, Qingdao 266042, PR China

$\dagger$ Electronic supplementary information (ESI) available. See DOI: 10.1039/c9ra04286e
}

the above NIR region and high photothermal conversion efficiency should be particularly emphasized. In the past decade, much effort has been devoted to engineer different kinds of photothermal agents, such as noble metal nanoparticles $(\mathrm{NPs}),{ }^{10,11}$ carbon nanotubes and derivative nanomaterials, ${ }^{12,13}$ transition metal sulfide NPs, ${ }^{14,15}$ and organic-based nanomaterials. ${ }^{16,17}$ Through controlling the morphology and surface chemistry, photothermal cancer therapy with high efficiency could be achieved.

Gold $(\mathrm{Au})$ nanomaterials especially those with anisotropic structures, such as Au nanorods, Au nanocages, Au nanostars and $\mathrm{Au}$ nanoshells, have played key roles in the development of PTT due to their facile preparation, excellent biocompatibility and controllable optical property of surface plasmon resonance (SPR) with high photothermal conversion efficiency. ${ }^{18-21}$ For instance, through optimizing the thickness of $\mathrm{Au}$ nanoshells and aspect ratio of Au nanorods, the SPR peak can be adjusted in NIR region to obtain optimal PTT effect. ${ }^{22-27}$ However, Au NPs with isotropic structures is not applicable for PTT since their optical absorption are mainly located in visible region. To adjust the SPR peak of Au NPs from visible to NIR region, one simple method was developed via the assembly of Au NPs assisted by the molecules with diverse chemical functionalities to generate the spectral coupling effects. Gao et al. have reported that the SPR peak shift of Au NPs to NIR regions for enhanced photothermal therapy could be successfully achieved by light-triggered 
cross-linking of Au NPs modified with photolabile diazirine moieties. ${ }^{28}$ Similarly, Chen et al. demonstrated their successful exploration on amphiphilicity-driven selfassembly of $\mathrm{Au}$ NPs modified with PEG- $b$-PCL blockcopolymer for further biological application. ${ }^{29,30}$ Though enhanced optical properties could be achieved by the assembly of Au NPs, the modification of Au NPs is complicated and the size of the hybrids is rarely controllable, which may hinder their extensive application in cancer therapy. Therefore, the development of Au NPs based photothermal agent with tunable optical properties by simple synthesis method is still of great importance.

In comparison with $\mathrm{Au}$ nanomaterials, platinum $(\mathrm{Pt})$ nanomaterials are identified as inactive optical element and have negligible absorption in NIR region. However, the growth of Pt NPs on the surface of Au nanostructures could effectively adjust the SPR behavior of Au nanostructures due to their surface plasmon coupling effect between Au core and Pt shell. ${ }^{31-35}$ For example, Pan et al. developed a two-step method for the synthesis of Au@Pt nanoparticles and applied them to cancer photothermal and radiation therapy taking advantage of the enhanced photothermal conversion and X-ray absorption. ${ }^{34}$ Chen et al. reported that the surface plasmonic behavior of Au nanorods could be easily tailored through the growth of ultrafine Pt NPs on the surface, resulting in red-shifting of the SPR band and elevated photothermal conversion efficiency. ${ }^{35}$ Apart from the improved optical properties, the stability of $\mathrm{Au}$ nanostructures has also been significantly enhanced after the growth of $\mathrm{Pt}$ NPs. ${ }^{36}$

In this paper, Au@Pt bimetallic NPs (Au@Pt NPs) with dendritic structure were synthesized through an ultrasound assisted one-step method in aqueous solution (Scheme 1). The absorption of Au@Pt NPs at $808 \mathrm{~nm}$ was obviously enhanced compared to Au NPs and could be easily regulated by the amount of Pt precursor. Au@Pt NPs exhibited excellent photostability with a high photothermal conversion efficiency of $44.2 \%$. After functionalized with biocompatible PEG, Au@Pt NPs presented a dose-dependent cellular killing capacity upon NIR laser irradiation, along with negligible cytotoxicity. Moreover, the potential application of Au@Pt NPs for PTT was demonstrated in xenograft tumor mouse model.

\section{Experimental section}

\subsection{Materials}

$\mathrm{HAuCl}_{4} \cdot 4 \mathrm{H}_{2} \mathrm{O}$ and $\mathrm{H}_{2} \mathrm{PtCl}_{6} \cdot 6 \mathrm{H}_{2} \mathrm{O}$ were purchased from Sinopharm Chemical Reagent Co. Ltd. Ascorbic acid (AA) and Pluronic F127 were purchased from Sigma. Methoxy-PEG-thiol $\left(M_{\mathrm{w}}\right.$ $=5000$ ) was purchased from Shanghai Tuo Yang Biotechnology Co. Ltd.

\subsection{Synthesis of Au@Pt NPs}

For the preparation of the Au@Pt NPs with different amount of Pt NPs, the molar ratios of Pt/Au were varied from 0.0 to 4.0

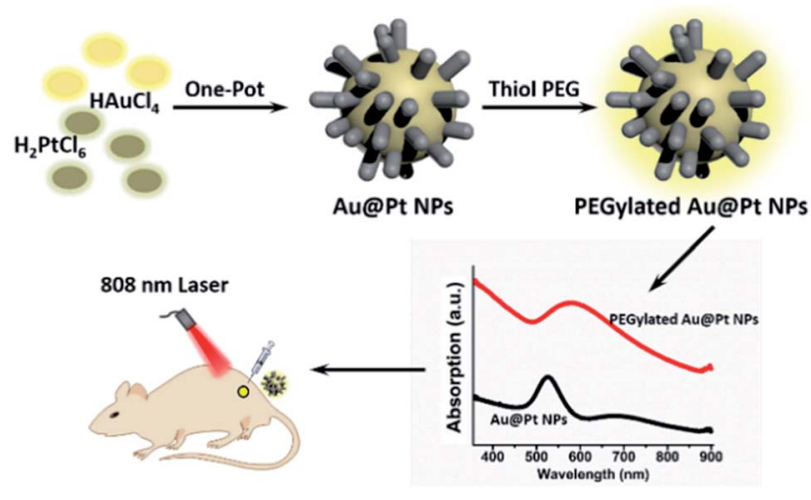

Scheme 1 Schematic illustration of Au@Pt NPs for photothermal cancer therapy.

in the aqueous solution. Typically, Pluronic F127 (0.03 g) was mixed with $\mathrm{H}_{2} \mathrm{PtCl}_{6} \cdot 6 \mathrm{H}_{2} \mathrm{O}$ solution $(20 \mathrm{mM}, 1.0 \mathrm{~mL})$ and $\mathrm{HAuCl}_{4} \cdot 4 \mathrm{H}_{2} \mathrm{O}$ solution $(20 \mathrm{mM}, 2.0 \mathrm{~mL})$ in a glass bottle and stirred to obtain homogeneous solution. Then, AA solution $(0.1 \mathrm{M}, 3.0 \mathrm{~mL})$ was added and sonicated for $20 \mathrm{~min}$, followed by gentle stirring for $24 \mathrm{~h}$ at room temperature. The final product was collected by centrifuging, washed with ethanol/ water for three times, and re-dispersed in water.

\subsection{PEGylation of Au@Pt nanoparticles}

Methoxy-PEG-thiol solution (3000 ppm, $3 \mathrm{~mL}$ ) was added into $\mathrm{Au} @ P t$ NPs solution (1000 ppm, $1 \mathrm{~mL}$ ) and sonicated for $10 \mathrm{~min}$ in ice. After stirring for $24 \mathrm{~h}$ at room temperature, the PEGylated $\mathrm{Au} @ P t$ NPs were separated by centrifuging at $12000 \mathrm{rpm}$ for $30 \mathrm{~min}$ and washed for three times to remove free methoxy-PEGthiol.

\subsection{Characterization}

Optical spectrum of Au@Pt NPs was monitored by a UV spectrophotometer (UV-2450, Shimadzu). X-ray diffraction spectra were performed on X-ray powder diffractometer (Ultima IV, Rigaku, Japan). Transmission electron microscopy images (TEM) were recorded on JEOL-1203 transmission electronic microscope (JEM-2010, Jeol, Japan). The average hydrodynamic diameter was determined by Dynamic Light Scattering (DLS, Nano-ZS90, Malvern). The concentrations of $\mathrm{Au}$ and $\mathrm{Pt}$ were analyzed by an inductively coupled plasma optical emission spectroscopy (ICP-OES, PerkinElmer Optima $5300 \mathrm{DV})$.

\subsection{Temperature measurement during NIR irradiation}

$\mathrm{Au}$ and Au@Pt NPs with different concentrations were irradiated by $808 \mathrm{~nm}$ laser with power density of $2 \mathrm{~W} \mathrm{~cm}^{-2}$ for $8 \mathrm{~min}$. The IR thermal imaging camera (FLIR) was used to record the changes of temperature with 0.5 or $1 \mathrm{~min}$ interval.

\subsection{Cell culture}

HeLa human cancer cells, 4T1 murine mammary carcinoma cells and CCRF-CEM human acute lymphatic leukemia cells 
were obtained from ATCC (American Type Culture Collection, Manassas, VA, USA). They were cultured in the media of RPMI1640 medium (Sigma, St. Louis, MO) with 10\% FBS and antibiotics at constant temperature of $37{ }^{\circ} \mathrm{C}$ with $5 \% \mathrm{CO}_{2}$.

\subsection{Cell viability assay}

Cell viability was determined by MTS assay, while the photothermal effect of Au and Au@Pt NPs were also evaluated by the viability of HeLa cells. Simply, HeLa cells seeded with $0.5 \times 10^{4}$ cells per well were incubated for $24 \mathrm{~h}$. Then, HeLa cells were divided into two groups: (1) control group without laser and (2) PTT group with laser irradiation. Subsequently, HeLa cells were cultivated with different concentrations of Au and Au@Pt NPs for $24 \mathrm{~h}$, then removing cell medium and following different treatments, with or without $808 \mathrm{~nm}$ laser irradiation $\left(1.0 \mathrm{~W} \mathrm{~cm}^{-2}, 6\right.$ min). Finally, the PTT effect was evaluated by measuring the absorbance at $490 \mathrm{~nm}$ with a microplate reader (BioTek).

To further evaluate the photothermal effect of Au@Pt NPs under laser irradiation in vitro by microscope, calcein-AM/ propidium iodide (PI) double staining experiment was performed. HeLa and CCRF-CEM cells were cultivated in optical cell culture dishes for $24 \mathrm{~h}$.

Then, Au@Pt NPs was added with final concentration of $150 \mathrm{ppm}$ and cultivated for $2 \mathrm{~h}$, followed by another $1 \mathrm{~h}$ cultivation under irradiation with $808 \mathrm{~nm}$ laser $\left(1.0 \mathrm{~W} \mathrm{~cm}^{-2}, 6 \mathrm{~min}\right)$. Finally, the cells were stained with both calcein-AM and PI to determine the photothermal effect of Au@Pt NPs by an inverted fluorescence microscope.

\subsection{Xenograft tumor mouse model and therapeutic evaluation}

Balb/c female mice (body weight $\sim 20 \mathrm{~g}$ ) were purchased from Hunan SLAC Laboratory Animal Co., Ltd. Mice bearing 4T1 tumors were divided into 4 groups with 6 mice per group: (a) control, (b) NIR only (1.5 W cm $\left.\mathrm{cm}^{-2}, 7 \mathrm{~min}\right)$, (c) Au@Pt NPs only (50 $\mu \mathrm{L}, 3000$ ppm), (d) Au@Pt NPs + NIR (50 $\mu \mathrm{L}, 3000$ ppm; $\left.1.5 \mathrm{~W} \mathrm{~cm}^{-2}, 7 \mathrm{~min}\right)$. Au@Pt NPs were directly injected to the tumors. All animal procedures were performed in accordance with the Guidelines for Care and Use of Laboratory Animals of Hunan University and experiments were approved by the Animal Ethics Committee of College of Biology (Hunan University).

\subsection{Hematoxylin and eosin (H\&E) histology analysis of tumor}

Mice were sacrificed at 30 days after PPT and the major organs (including liver, spleen, heart, lung and kidney) were collected and fixed in $4 \%$ paraformaldehyde. After dehydrated in different concentration sucrose solution, small pieces of organs were fixed, sectioned and stained with H\&E.

\section{Results and discussion}

\subsection{Synthesis and characterization of Au@Pt NPs}

The strategy used to synthesize the nanoparticles was according to the previously reported method. ${ }^{37-40} \mathrm{HAuCl}_{4}$ and
$\mathrm{H}_{2} \mathrm{PtCl}_{6}$ were chosen as the metal precursor because they have different standard reduction potentials to realize sequential reduction of different precursor in one pot. Experimentally, Au@Pt NPs were synthesized by direct reducing the mixed precursor solution of $\mathrm{Au}$ and $\mathrm{Pt}$ (the molar ratio of $\mathrm{Pt} / \mathrm{Au}$ is 2) assisted by ultrasound. As shown in Fig. 1a and b, Au@Pt NPs exhibit uniform branched structures with sizes ranging from 20 to $30 \mathrm{~nm}$. Whereas, the increasing or decreasing the proportion of Pt could lead to incomplete coverage or selfnucleation of Pt (Fig. S1 $\dagger$ ). To better observe the clear morphology of Au@Pt NPs, high-angle annular dark-field scanning transmission electron microscopy (HAADF-STEM), and energy dispersive X-ray (EDX)-elemental mapping were carried out. As revealed in Fig. 1c, Au element is mainly located in the center of Au@Pt NPs and surrounded by multiple Pt NPs, which is well consistent with the different contrasts in the TEM images. In addition, the XRD pattern confirm the coexistence of $\mathrm{Au}$ and Pt NPs in Au@Pt NPs.

Considering the SPR effect of the Au core and Pt shell, the absorption spectrum of Au@Pt NPs was monitored by a UV-Vis spectrophotometer, as shown in Fig. 1e. Meanwhile, time tracking of optical property of Au@Pt NPs (Fig. S2 $\dagger$ ) and the standard curve of Au@Pt NPs (Fig. S3 $\dagger$ ) were tested. As the Pt/ $\mathrm{Au}$ ratio increased from 0 to 2 (Fig. $\mathrm{S} 4 \dagger$ ), the SPR band exhibits dose- and time-dependent SPR red-shifting. The relevant absorption intensity at $808 \mathrm{~nm}$ of Au@Pt NPs and Au NPs against increasing concentration is plotted in Fig. 1f. Interestingly, the absorption at 808 nm of Au@Pt NPs is 8 times that of pure $\mathrm{Au}$ NPs at the same Au concentration, further confirming the excellent NIR absorption property of Au@Pt NPs. Then, in order to improve their biocompatibility and physiological stability, methoxy-PEG-thiol was introduced for surface modification of Au@Pt NPs. The hydrodynamic size of the PEGylated Au@Pt NPs was about $110 \mathrm{~nm}$ as determined by DLS, larger than that of measured by TEM (Fig. S5 $\dagger$ ), which is consistent with the previously published work. Additionally, PEGylated Au@Pt NPs displayed excellent dispersibility in
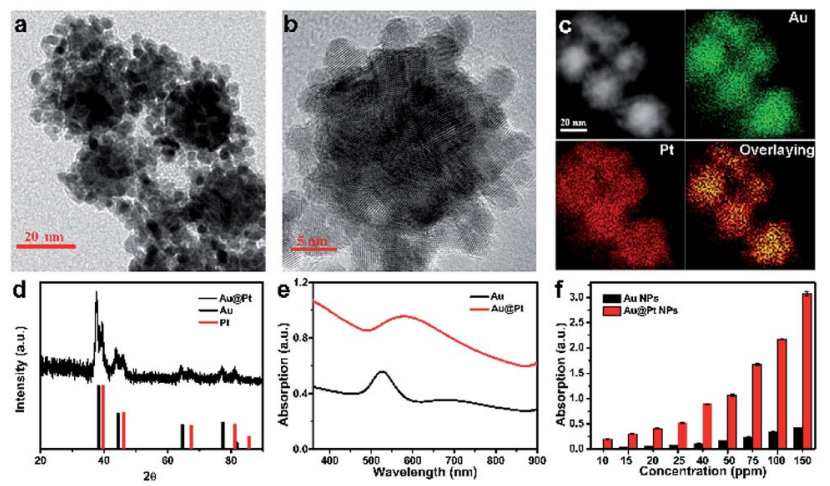

Fig. 1 Synthesis and characterizations of Au@Pt NPs. (a and b) Representative TEM images with different magnifications. (c) HAADFSTEM image and EDX mapping images of AuCPt NPs. (d) XRD pattern of Au@Pt NPs. (e) UV-Vis absorption spectra of Au and Au@Pt NPs. (f) The absorption at $808 \mathrm{~nm}$ of Au NPs and Au@Pt NPs with the same concentration of Au element. 
water, Dulbecco phosphate-buffered saline (DPBS) buffer and serum without notable aggregation in $48 \mathrm{~h}$ (Fig. S6†).

\subsection{Photothermal conversion effect of Au@Pt nanoparticles}

Inspired by the coupling effect of Pt NPs on the SPR of Au@Pt NPs, photothermal effect of Au@Pt NPs upon the irradiation of $808 \mathrm{~nm}$ laser was investigated as shown in Fig. 2a and b. Au NPs and Au@Pt NPs both exhibit dose-dependent heating capacity for the absorption increases with concentration. It's worth mentioning that Au@Pt NPs solution could reach higher temperatures than Au NPs with the same amount of $\mathrm{Au}$, contributed by the SPR red-shifting of Au NPs. For example, with the $\mathrm{Au}$ concentration of $100 \mathrm{ppm}$, the temperature of the solution increased by $33.8^{\circ} \mathrm{C}$ (from $25.6{ }^{\circ} \mathrm{C}$ to $59.4{ }^{\circ} \mathrm{C}$ ) under 8 min irradiation, about three-fold compared to that of $\mathrm{Au}$ NPs, indicating the high photothermal conversion effect of Au@Pt NPs. Meanwhile, Au@Pt NPs exhibited excellent photothermal effects in physiological conditions as shown in Fig. S7. $\uparrow$ Then, we quantitatively studied the photothermal conversion efficiency of Au@Pt NPs following the calculation principle from Roper's methods. ${ }^{41,42}$ Thus, the heat conversion efficiency $(\eta)$ of the Au@Pt NPs under $808 \mathrm{~nm}$ laser can be calculated to be about $44.2 \%$ (Fig. S8 $\dagger$ ), which is higher than that of most reported study, such as Au nanorods (21\%), ${ }^{43}$ $\mathrm{Bi}_{2} \mathrm{Se}_{3}(26.4 \%)^{44}$ and PEGylated black phosphorous (BP) $(27.4 \%){ }^{45}$

After demonstrating the excellent heating capacity of $\mathrm{Au} @ P t$ NPs, the photostability of Au@Pt NPs were evaluated in detail, as shown in Fig. $2 \mathrm{c}$ and d. $1 \mathrm{~mL}$ of Au@Pt NPs solution with a concentration of $50 \mathrm{ppm}$ was exposed to NIR laser for four on/off cycles and each cycle lasts for $20 \mathrm{~min}$.
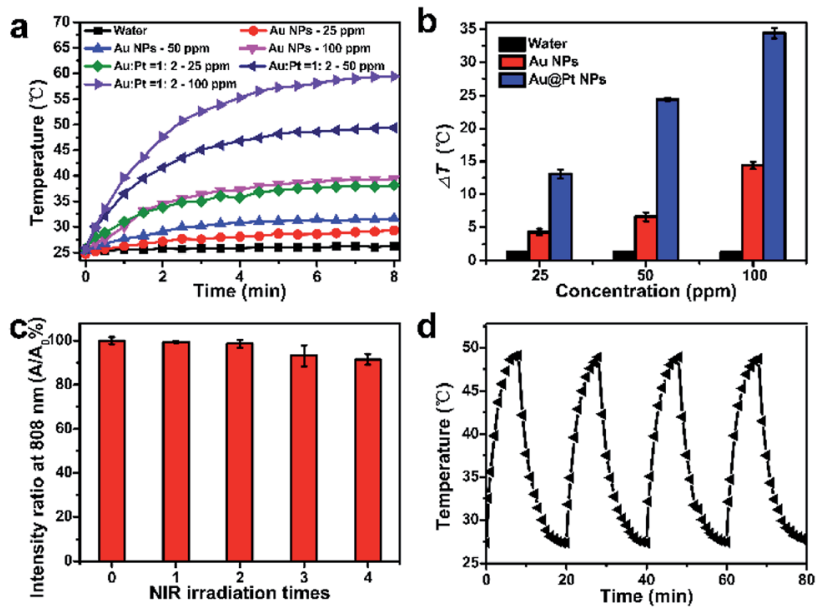

Fig. 2 (a) The temperature changes of different concentration of $\mathrm{Au}$ NPs and Au@Pt NPs under an $808 \mathrm{~nm}$ laser irradiation. (b) The temperature change $(\Delta T)$ under an $808 \mathrm{~nm}$ laser irradiation with distilled water, different concentrations of Au NPs and Au@Pt NPs. (c) Variation of the absorption ratios at $808 \mathrm{~nm}\left(A / A_{0} \%\right)$ of Au@Pt NPs after different irradiation cycles. (d) The time-dependent temperature curves of Au@Pt NPs with an 808 nm NIR laser for four on/off cycles.
During the heating/cooling processing, the temperature and absorption at $808 \mathrm{~nm}$ were recorded, respectively. Obviously, the absorption at $808 \mathrm{~nm}$, the heating curves, and maximum temperature were maintained the same after four on/off heating/cooling cycles, revealing the excellent photostability of Au@Pt NPs. On the contrary, a marked decline in absorption ( $c a .40 \%$ ) was observed in the case of $\mathrm{Au}$ nanorods with the same Au concentration (Fig. S9†) due to the inevitable morphological changes of $\mathrm{Au}$ nanorods nanostructure upon NIR irradiation, ${ }^{46-48}$ which further indicates the outstanding photostability of Au@Pt NPs. Taken together, Au@Pt NPs exhibited enhanced NIR light absorption and photothermal conversion properties, as well as excellent photostability, suggesting great potential as an ideal photothermal treatment agent.

\subsection{In vitro cytotoxicity and photothermal cytotoxicity}

Since biocompatibility of nanomaterials is an important prerequisite for their application in cancer therapy, the cell viability after incubation with Au@Pt NPs was tested using a standard MTS assay, as shown in Fig. 3a. After incubation for $24 \mathrm{~h}, \mathrm{Au} @ P t$ NPs did not cause any significant cytotoxicity in HeLa cells at tested concentrations, demonstrating the excellent biocompatibility of Au@Pt NPs at cellular level. This result further drove us to explore the PTT effect of Au@Pt NPs on HeLa
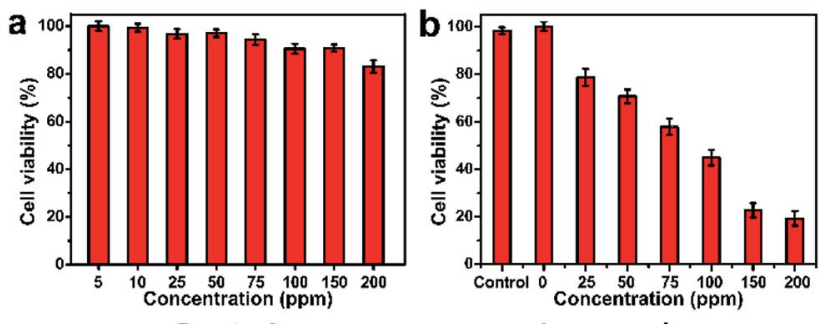

C
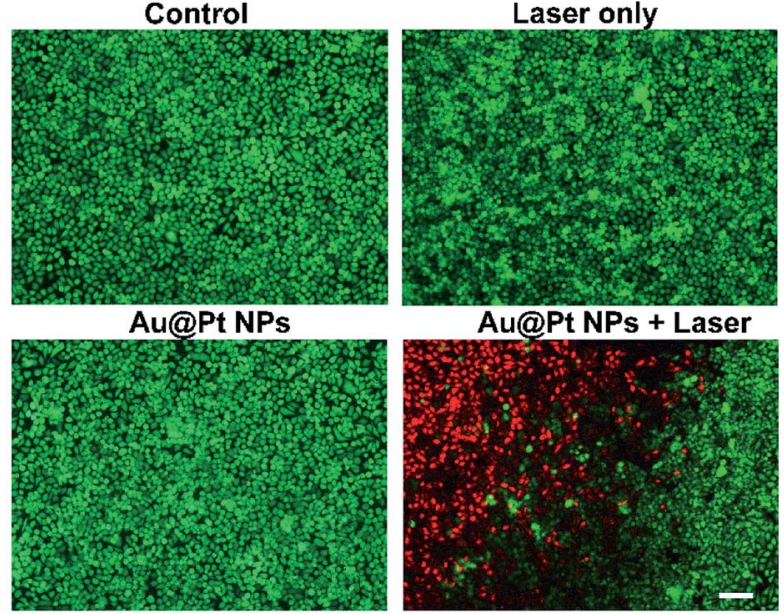

Fig. 3 (a) Cytotoxicity of AuQPt NPs with different concentrations after incubated with HeLa cells for $24 \mathrm{~h}$. (b) Cell viability of HeLa cells treated with Au@Pt NPs (200 ppm) under NIR laser irradiation $(808 \mathrm{~nm}$, $1 \mathrm{~W} \mathrm{~cm}{ }^{-2}$ ) for $6 \mathrm{~min}$. (c) Fluorescent images of HeLa cells stained with Live/Dead Double Stain Kit, green fluorescence indicates live cells by calcein-AM, and red fluorescence indicates dead cells by PI. Scale bar, $100 \mu \mathrm{m}$. 

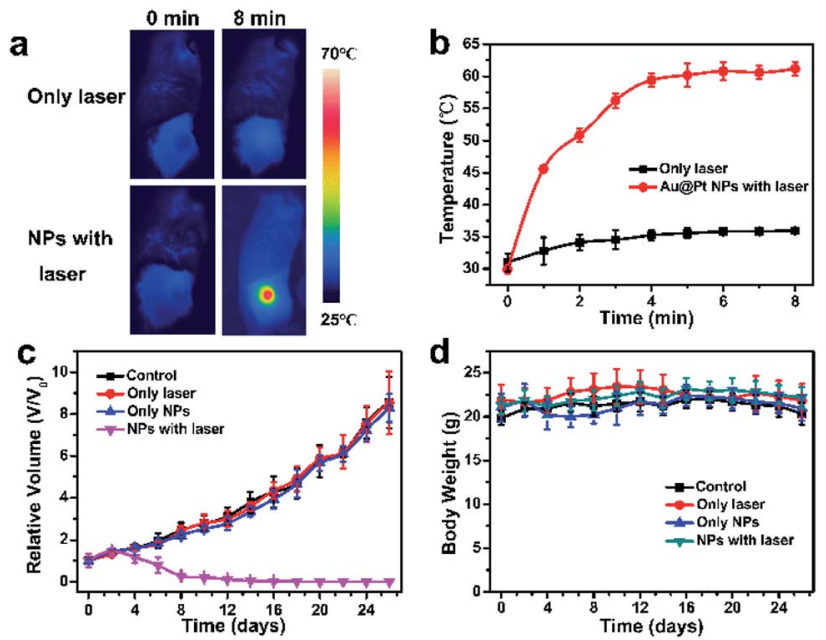

Fig. 4 (a) IR thermal images of 4T1-tumor-bearing mice with or without Au@Pt NPs injection under $808 \mathrm{~nm}$ laser irradiation. (b) The tumor temperature changes based on IR thermal imaging during laser irradiation. (c) Relatively tumor volume of four groups with different treatments. The tumor volume is normalized to their initial sizes. (d) The Body weight curves of four groups with different treatments.

cells under NIR laser irradiation. As shown in Fig. 3b, a dosedependent cell killing efficiency was presented, and less than $20 \%$ of cells survived after treatment at a concentration of $150 \mathrm{ppm}$ or higher, demonstrating the outstanding therapeutic efficacy of Au@Pt NPs-based PTT.

On the other hand, to compare the PTT effect of Au@Pt NPs and Au NPs in vitro, the PTT effect of Au and Au@Pt NPs with the same concentration of Au element was also examined (Fig. S10†). As expected, Au NPs also exhibited excellent biocompatibility as Au@Pt NPs. However, the PTT effect of Au NPs was much lower than that of Au@Pt NPs, which further demonstrate the enhanced optical and photothermal properties of Au@Pt NPs. Live/dead double staining of HeLa and CCRF-CEM cells by calcein-AM/PI stain kit were also carried out for direct observation of the cytotoxicity and PTT effect (Fig. $3 \mathrm{c}$ and S11†). Compared with control group, cells treated with only laser and only Au@Pt NPs exhibited negligible cytotoxicity caused by laser and Au@Pt NPs. After incubation with Au@Pt NPs, part of the cells was irradiated by NIR laser. Obvious boundary between red fluorescence and green fluorescence could be seen on the edge of the laser spot. From all the above experiments, the therapeutic characteristics of $\mathrm{Au} @ P t$ NPs provide great potential for the enhanced PTT in vitro.

\subsection{In vivo photothermal therapy}

4T1 tumor-bearing Balb/c mice were employed as xenograft models to determine the PTT effect of Au@Pt NPs in vivo. Mice bearing tumor were randomly divided into four groups: (1) group with only PBS injection, (2) group with PBS injection and laser irradiation, (3) group with only Au@Pt NPs injection and (4) group with Au@Pt NPs injection and laser irradiation. The real-time temperature changes of the tumor sites of mice upon laser irradiation were detected by an IR thermal camera. The temperature of tumor sites injected with Au@Pt NPs rose rapidly and could reach as high as $60^{\circ} \mathrm{C}$ in 4 min under the NIR laser irradiation, resulting in significant histological damage in tumor sections. While, there were no obvious temperature rise or tissue damage for those treated with only laser (increased less than $5{ }^{\circ} \mathrm{C}$ ) after the same irradiation period (Fig. 4a, b and S12†). Significant tumor growth suppression could be observed in groups with Au@Pt NPs injection under NIR laser irradiation while those control groups showed rapid tumor growth during the 16 days period (Fig. 4c). In addition, the cured mice were kept for another 30 days and no any indications of tumor recurrence could be observed.

\subsection{In vivo toxicity study}

Generally, the body weight of the mice during the treatment period is a key criterion to evaluate the potential toxicity of nanomaterials in vivo. ${ }^{49}$ The body weight of mice remained similar in all groups during the treatment period (Fig. 4d), indicating the high biosafety of Au@Pt NPs. Moreover, the major organs of the mice in key groups were collected for histological analysis after the mice sacrificed from the treatment. As shown in Fig. 5, no significant organ damage could be found in different groups after PTT treatment, demonstrating no obvious side effects of Au@Pt NPs in vivo.

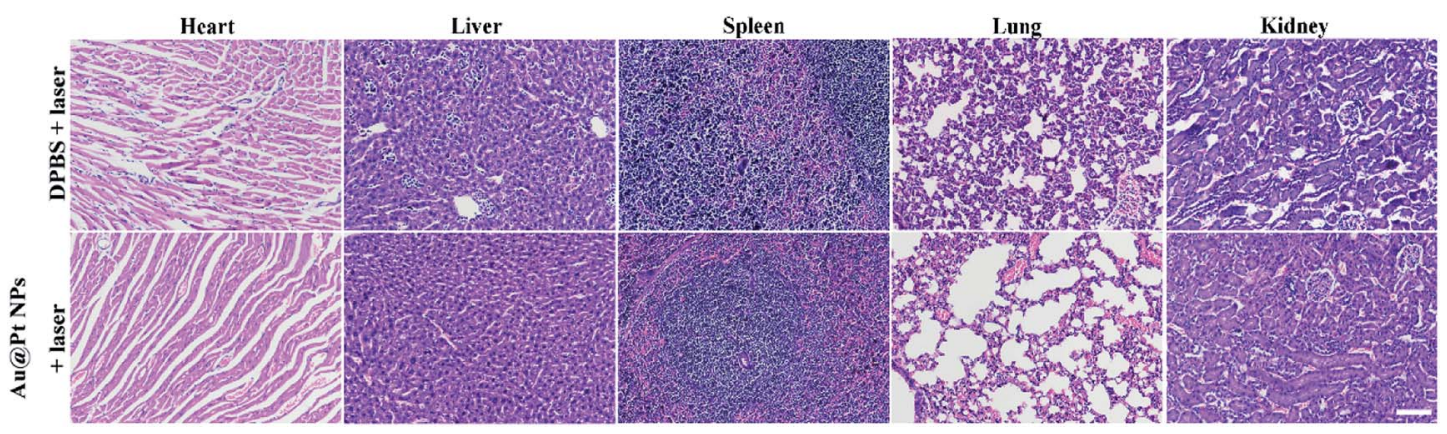

Fig. 5 H\&E-stained tissue sections of major organs harvested from mice with different treatments. Scale bar, $50 \mu \mathrm{m}$. 


\section{Conclusion}

In summary, we have successfully synthesized Au@Pt NPs with dendritic structure through an ultrasonic assisted one-step method in aqueous solution. The optical properties of Au@Pt NPs could be easily manipulated by tailoring the SPR coupling effect between the Au core and Pt shell. The absorption of $\mathrm{Au} @ P t$ NPs at $808 \mathrm{~nm}$ was obviously enhanced compared to Au NPs, 8 times that of pure Au NPs at the same Au concentration. Au@Pt NPs exhibited excellent photostability with a high photothermal conversion efficiency of $44.2 \%$ under $808 \mathrm{~nm}$ light irradiation. By introducing methoxy-PEG-thiol, the biocompatibility and physiological stability were largely improved. Besides negligible cytotoxicity, Au@Pt NPs possess outstanding cellular killing capacity upon NIR laser irradiation with a dosedependent manner. The in vitro experiments showed the PTT efficiency of Au@Pt NPs was much higher than that of Au NPs, which was consistent with the enhanced SPR absorption properties of Au@Pt NPs. Moreover, the potential application of $\mathrm{Au} @ P t$ NPs for PTT in vivo was successfully carried out in xenograft tumor mouse model and effective inhibition on tumor growth was obtained. Overall, Au@Pt NPs have shown great potential as new photothermal agent in the battle against cancer and the simple synthesis method will provide new ideas for the field of bionanomaterials.

\section{Conflicts of interest}

There are no conflicts to declare.

\section{Acknowledgements}

This work was financially supported by the NSFC (21705037, 21622504, 21735001, and 21877029), Hunan Provincial Natural Science Foundation of China (2018JJ3092), the Science and Technology Project of Hunan Province (2017RS3019), the Open Funding Project of the State Key Laboratory of Bioreactor Engineering and the Fundamental Research Funds for the Central Universities.

\section{References}

1 X. Deng, K. Li, X. Cai, B. Liu, Y. Wei, K. Deng, Z. Xie, Z. Wu, P. a. Ma, Z. Hou, Z. Cheng and J. Lin, Adv. Mater., 2017, 29, 1701266.

2 Q. Dong, X. Wang, X. Hu, L. Xiao, L. Zhang, L. Song, M. Xu, Y. Zou, L. Chen, Z. Chen and W. Tan, Angew. Chem., Int. Ed., 2018, 57, 1433-7851.

3 S. Eustis and M. A. El-Sayed, Chem. Soc. Rev., 2006, 35, 209217.

4 X. Huang, P. K. Jain, I. H. El-Sayed and M. A. El-Sayed, Lasers Med. Sci., 2007, 23, 217.

5 S. Lal, S. E. Clare and N. J. Halas, Acc. Chem. Res., 2008, 41, 1842-1851.

6 F. Yang, A. Skripka, M. S. Tabatabaei, S. H. Hong, F. Ren, A. Benayas, J. K. Oh, S. Martel, X. Liu, F. Vetrone and D. Ma, ACS Nano, 2019, 13, 408-420.
7 F. Yang, A. Skripka, M. S. Tabatabaei, S. H. Hong, F. Ren, Y. Huang, J. K. Oh, S. Martel, X. Liu, F. Vetrone and D. Ma, Chem. Mater., 2019, 31, 3201-3210.

8 F. Yang, A. Skripka, A. Benayas, X. Dong, S. H. Hong, F. Ren, J. K. Oh, X. Liu, F. Vetrone and D. Ma, Adv. Funct. Mater., 2018, 28, 1706235.

9 F. Ren, B. del Rosal, S. Y. An, F. Yang, E. Carrasco, A. Benayas, J. K. Oh, D. Jaque, Á. J. de la Fuente, F. Vetrone and D. Ma, Part. Part. Syst. Charact., 2017, 34, 1600242.

10 P. K. Jain, X. Huang, I. H. El-Sayed and M. A. El-Sayed, Acc. Chem. Res., 2008, 41, 1578-1586.

11 M. Chen, S. Tang, Z. Guo, X. Wang, S. Mo, X. Huang, G. Liu and N. Zheng, Adv. Mater., 2014, 26, 8210-8216.

12 K. Yang, S. Zhang, G. Zhang, X. Sun, S.-T. Lee and Z. Liu, Nano Lett., 2010, 10, 3318-3323.

13 C. Liang, S. Diao, C. Wang, H. Gong, T. Liu, G. Hong, X. Shi, H. Dai and Z. Liu, Adv. Mater., 2014, 26, 5646-5652.

14 S. Wang, X. Li, Y. Chen, X. Cai, H. Yao, W. Gao, Y. Zheng, X. An, J. Shi and H. Chen, Adv. Mater., 2015, 27, 2775-2782.

15 Y. Liu, X. Ji, J. Liu, W. W. L. Tong, D. Askhatova and J. Shi, Adv. Funct. Mater., 2017, 27, 1703261.

16 M. Chen, X. Fang, S. Tang and N. Zheng, Chem. Commun., 2012, 48, 8934-8936.

17 C. Wang, H. Xu, C. Liang, Y. Liu, Z. Li, G. Yang, L. Cheng, Y. Li and Z. Liu, ACS Nano, 2013, 7, 6782-6795.

18 M. R. K. Ali, S. R. Panikkanvalappil and M. A. El-Sayed, J. Am. Chem. Soc., 2014, 136, 4464-4467.

19 D. E. Kruse, D. N. Stephens, H. A. Lindfors, E. S. Ingham, E. E. Paoli and K. W. Ferrara, IEEE Trans. Biomed. Eng., 2011, 58, 2002-2012.

20 J. Wang, M. You, G. Zhu, M. I. Shukoor, Z. Chen, Z. Zhao, M. B. Altman, Q. Yuan, Z. Zhu, Y. Chen, C. Z. Huang and W. Tan, Small, 2013, 9, 3678-3684.

21 N. Wang, Z. Zhao, Y. Lv, H. Fan, H. Bai, H. Meng, Y. Long, T. Fu, X. Zhang and W. Tan, Nano Res., 2014, 7, 1291-1301.

22 M. R. K. Ali, M. A. Rahman, Y. Wu, T. Han, X. Peng, M. A. Mackey, D. Wang, H. J. Shin, Z. G. Chen, H. Xiao, R. Wu, Y. Tang, D. M. Shin and M. A. El-Sayed, Proc. Natl. Acad. Sci., 2017, 114, E3110-E3118.

23 S. Gao, L. Zhang, G. Wang, K. Yang, M. Chen, R. Tian, Q. Ma and L. Zhu, Biomaterials, 2016, 79, 36-45.

24 R. Xing, K. Liu, T. Jiao, N. Zhang, K. Ma, R. Zhang, Q. Zou, G. Ma and X. Yan, Adv. Mater., 2016, 28, 3669-3676.

25 L. Li, S. Fu, C. Chen, X. Wang, C. Fu, S. Wang, W. Guo, X. Yu, X. Zhang, Z. Liu, J. Qiu and H. Liu, ACS Nano, 2016, 10, 70947105.

26 J. Song, X. Yang, Z. Yang, L. Lin, Y. Liu, Z. Zhou, Z. Shen, G. Yu, Y. Dai, O. Jacobson, J. Munasinghe, B. Yung, G.-J. Teng and X. Chen, ACS Nano, 2017, 11, 6102-6113.

27 Y. Liu, Z. Wang, Y. Liu, G. Zhu, O. Jacobson, X. Fu, R. Bai, X. Lin, N. Lu, X. Yang, W. Fan, J. Song, Z. Wang, G. Yu, F. Zhang, H. Kalish, G. Niu, Z. Nie and X. Chen, ACS Nano, 2017, 11, 10539-10548.

28 X. Cheng, R. Sun, L. Yin, Z. Chai, H. Shi and M. Gao, Adv. Mater., 2017, 29, 1604894. 
29 P. Huang, J. Lin, W. Li, P. Rong, Z. Wang, S. Wang, X. Wang, X. Sun, M. Aronova, G. Niu, R. D. Leapman, Z. Nie and X. Chen, Angew. Chem., Int. Ed., 2013, 125, 14208-14214.

30 J. Song, P. Huang, H. Duan and X. Chen, Acc. Chem. Res., 2015, 48, 2506-2515.

31 Y. Fu, Q. Feng, Y. Chen, Y. Shen, Q. Su, Y. Zhang, X. Zhou and Y. Cheng, Mol. Pharm., 2016, 13, 3308-3317.

32 M. Grzelczak, J. Pérez-Juste, F. J. García de Abajo and L. M. Liz-Marzán, J. Phys. Chem. C, 2007, 111, 6183-6188.

33 W. He, Y. Liu, J. Yuan, J.-J. Yin, X. Wu, X. Hu, K. Zhang, J. Liu, C. Chen, Y. Ji and Y. Guo, Biomaterials, 2011, 32, 1139-1147.

34 X. Liu, X. Zhang, M. Zhu, G. Lin, J. Liu, Z. Zhou, X. Tian and Y. Pan, ACS Appl. Mater. Interfaces, 2017, 9, 279-285.

35 J. Tang, X. Jiang, L. Wang, H. Zhang, Z. Hu, Y. Liu, X. Wu and C. Chen, Nanoscale, 2014, 6, 3670-3678.

36 H.-J. Jang, S. Hong, S. Ham, K. L. Shuford and S. Park, Nanoscale, 2014, 6, 7339-7345.

37 L. Yang, J. Chen, X. Zhong, K. Cui, Y. Xu and Y. Kuang, Colloids Surf., A, 2007, 295, 21-26.

$38 \mathrm{H}$. Ataee-Esfahani, L. Wang and Y. Yamauchi, Chem. Commun., 2010, 46, 3684-3686.

39 W. Shuangyin, K. Noel, J. Sanping and W. Xin, Nanotechnology, 2009, 20, 025605.
40 S. Guo, J. Li, S. Dong and E. Wang, J. Phys. Chem. C, 2010, 114, 15337-15342.

41 F. Gao, M. Sun, L. Xu, L. Liu, H. Kuang and C. Xu, Adv. Funct. Mater., 2017, 27, 1700605.

42 S. A. Chechetka, Y. Yu, X. Zhen, M. Pramanik, K. Pu and E. Miyako, Nat. Commun., 2017, 8, 15432.

43 D. K. Roper, W. Ahn and M. Hoepfner, J. Phys. Chem. C, 2007, 111, 3636-3641.

44 Z. Li, J. Shao, Q. Luo, X.-F. Yu, H. Xie, H. Fu, S. Tang, H. Wang, G. Han and P. K. Chu, Biomaterials, 2017, 133, 37-48.

45 C. Sun, L. Wen, J. Zeng, Y. Wang, Q. Sun, L. Deng, C. Zhao and Z. Li, Biomaterials, 2016, 91, 81-89.

46 Y. Liu, E. N. Mills and R. J. Composto, J. Mater. Chem. A, 2009, 19, 2704-2709.

47 H. Petrova, J. Perez Juste, I. Pastoriza-Santos, G. V. Hartland, L. M. Liz-Marzán and P. Mulvaney, Phys. Chem. Chem. Phys., 2006, 8, 814-821.

48 J. Wei, C. Kan, Y. Lou, Y. Ni, H. Xu and C. Wang, Superlattices Microstruct., 2016, 100, 315-323.

49 H.-W. Yang, Biomaterials, 2013, 34, 7204-7214. 